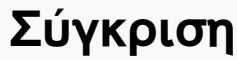

Tó .7 (1996)

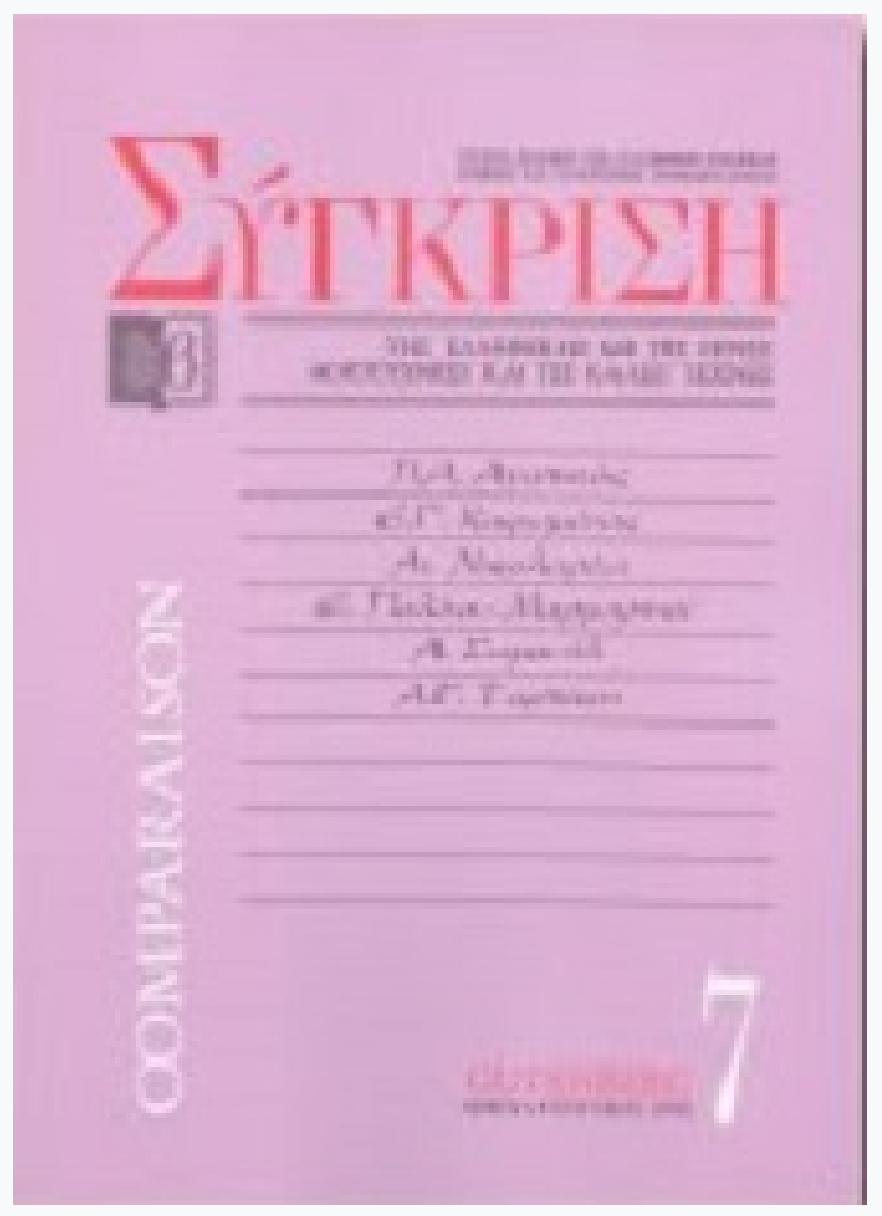

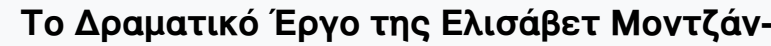
Maptıv́үкоu

Anna Tabaki

doi: $10.12681 /$ comparison.10740

Copyright $\odot$ 2016, Anna Tabaki

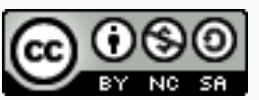

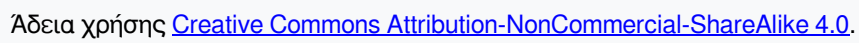

\section{Bıßлıорафıкń avaчopá:}

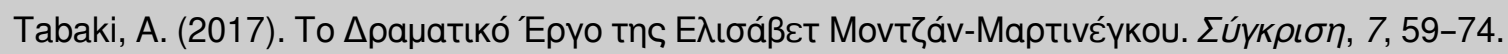

https://doi.org/10.12681/comparison.10740 


\section{L'œuvre dramatique d'Élisabeth Moutzan-Martinengou*}

LISABETH MOUTZAN-MARTINENGOU (ZANTE, 1801-1832), SECONDE FILle de François Moutzan et d'Angélique, née Sigourou, naît de deux familles de souche ancienne et noble. D'origine vénitienne, la famille des Moutzan - dont le père d'Élisabeth, François, fut un des members les plus brillants, ayant occupé de hautes charges administratives et politiques-, apparaît en effet dans le Livre d'or dès 1633 . $^{1}$

C'est à travers son Autobiographie, ${ }^{2}$ texte traversé d'une spontanéité très émouvante et considéré comme d' "une qualité litté- raire exceptionnelle" qui se range parmi "les ornements les plus purs de notre littérature grecque moderne" ${ }^{3}$ que nous pouvons saisir le caractère presque spasmodique et interrompu à maintes reprises de son éducation, le climat régnant dans la maison familiale, les possibilités d'information culturelle d'Élisabeth, par le biais notamment de la bibliothèque paternelle, ainsi que les étapes progressives de son évolution spirituelle, en l'occurrence dramaturgique. En outre, et malgré le fait que les écrits autobiographiques de la noble zantiote furent réhabilités ces dernières années, évalués surtout du point de vue de la formulation sous-jacente d'un message d'émancipation féminine, ${ }^{4}$ et par rapport aux idées rénovatrices apportées dans ce domaine par l'Aufklärung, la question concernant l'analyse de son ouvre créative n'a pas été abordée.

Notons qu'en effet son Autobiographie s'appuie sur quelques notions fondamentales de l'esprit des Lumières: le dépassement des passions humaines par la raison, la recherche de la vertu, la défense des droits de

* Cf. Anna TABAKI, Le théâtre néohellénique. Genèse et formation. Ses composantes sociales, idéologiques et esthétiques, thèse de docrorat, Paris, EHESS, 1995, vol. I. pp. I8-38. 
l'homme et ceux de liberté nationale, la foi en la force de l'enseignement ainsi que dans le profit apporté par l'éducation à la société, la fusion de la littérature avec la philosophie et les sciences, enfin, le sentiment de contemplation, l'amour ressenti pour la nature. ${ }^{5}$

L'inclination d'Élisabeth, son penchant très fort pour les lettres, imprègne ses écrits autobiographiques. Tout d'abord, ce furent sa mère et sa grandmère qui lui apprirent à lire et à écrire. Vue l'obstination de la jeune fille, sa soif d'apprendre, sa famille décida plus tard d'engager quelques précepteurs, tour à tour le prêtre Georges Tsoukalas, ${ }^{6}$ Basile Romantzas qui avait poursuivi des études à Padoue et a ultérieurement développé une activité de prédicateur et d'enseignant à Constantinople ${ }^{7}$ et, enfin, Théodore Dimadis, ayant poursuivi des cours de philosophie à l'Université de Pise: à l'époque où il fréquentait la maison des Moutzan, il avait justement été chargé des cours de philosophie à l'École publique de Zante. ${ }^{8}$ Dimadis semble avoir considérablement influencé son élève, en aiguisant sa conscience nationale tout en lui communiquant son enthousiasme pour les événements révolutionnaires survenus en Grèce. Néanmoins, ces enseignements, de courte durée et de nature plutôt circonstancielle, n'acquirent point un caractère systématique. Élisabeth fut quasiment, dans les grandes lignes, une autodidacte. Elle décrit par exemple comment elle a appris toute seule l'italien en utilisant l'édition bilingue du livre populaire Fior di Virtù-'Av$\theta 0 \varsigma \chi \alpha$ ei $\tau \omega v,{ }^{9}$ aussi bien que, plus tard, le français. ${ }^{10}$ Ainsi parmi ses lectures, pouvons-nous repérer quelques titres: outre, certains ouvrages historiques en italien et en français auxquels elle fait allusion, nous retenons le Magasin

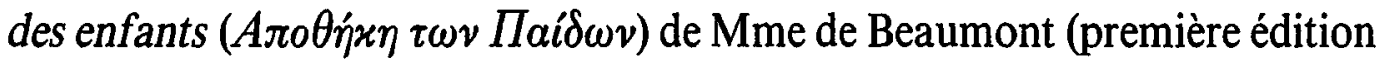

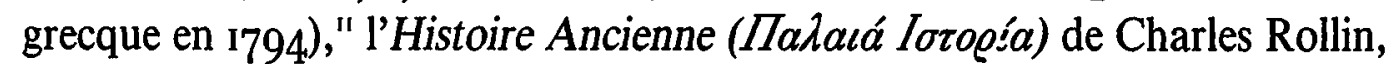
transposée en grec par Alexandre Canghellarios, en $175^{12}$ ainsi qu'un

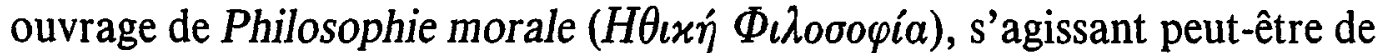
la traduction de Muratori, publiée par J. Moesiodax, en ${ }_{17} 60 .{ }^{13}$ A l'âge de I9 ans, Élisabeth composa un dialogue moral en italien, intitulé "Emilia ed Etelvige ovvero dell'invidia", qu'elle a intégralement recopié dans son Autobiographie. Jeune écrivain, elle attribue une valeur absolue à la conquête de la vertu intérieure ainsi qu'à la lutte permanente contre les passions négatives, les vices, que celle-là présuppose. C'est exactement ce processus de combat intérieur qui permettra à l'héroîne, dévorée par les vices de la jalousie et de l'avidité, lutter contre eux, afin de pouvoir vivre harmo60 nieusement en société. C'est juste après que l'heure de la première composition dramatique arriva: 
A la suite de ce dialogue, sans avoir lu la moindre règle poétique, j'ai voulu écrire une tragédie. J'en ai trouvé le sujet avec beaucoup de facilité, je l'ai rédigée en italien et en prose mais comme je l'ai composée sans connaître les normes de l'art dramatique, elle ressemble davantage à un roman qu'à une tragédie. Cependant, son sujet -fondé sur le principe de la v ra is e m$\mathrm{b}$ l a n ce- est $\mathrm{tr}$ è $\mathrm{s}$ mor a l et $\mathrm{t} r$ ès $\mathrm{p}$ a t hét i que, puisqu'il démontre que la Providence sauvegarde les innocents et ne les laisse pas être injustement tourmentés; je l'ai donc intitulée Enrico o l'innocenza. ${ }^{14}$

Selon l'aveu d'Élisabeth, la qualité du texte surprend très agréablement son maître Basile Romantzas, qui y rencontre avec plaisir de nombreuses opinions morales et l'incite à continuer dans cette voie.

A partir de ce moment, les questions d'ordre théorique commencent à préoccuper sérieusement le jeune auteur, questions qu'elle se mettra à résoudre au fur et à mesure de ses lectures de nature esthétique. Parallèlement, elle s'adonna régulièrement à ses ébauches littéraires. Au cours de l'année i820, elle informe qu'elle est désormais en mesure de connaître les règles de la Poétique. Cependant, elle note que, dans sa tragédie intitulée "Teano o la Giustizia legale", elle a presqu'instinctivement respecté, les règles des trois unités, à savoir d'action, de temps et de lieu. Elle a peint des caractères fermes, inébranlables ( $\alpha \sigma a ́ \lambda \varepsilon v \tau o \iota)$ et l'action est pathétique et morale. Poussée pour ainsi dire par un élan naturel, elle a composé, presque à son insu, une tragédie régulière dans les règles de l'art!'s Voilà comment Élisabeth expose l'intrigue de la tragédie, articulée autour du conflit entre les deux fils de la Reine de Macédoine:

Teano. Reina di Macedonia, vedendo il suo regno assediato da'Thessali, possenti nemici, divise le sue genti in due parti e fece dell'una generale il suo figlio Abante e all'altra Euridamante, uomo di singolare valore, e con simil numero di gente, andarono questi due generali a combattere. Euridamente appena sconfitto il poderoso esercito de'Thessali fu chiamato d'Abante in aiuto, il quale si vedea in procinto di perdersi con tutta la sua gente. Giunto dunque il valoroso Euridamante, il trasse dalla morte e mise in rotta le falangi de'Thessali. L'ingrato Abante invece di ringraziare il suo benefattore, se ne avvelenò d'invidia a cagione delle di lui prodezze e per soddisfare alla sua passione il fece comparire traditore. Il prode Euridamante invece di vedersi premiato per le sue vittorie, si vide carico di cateme, invece d'esser condotto al talamo di Adelaide - che gli fu promessa dalla reina sua madre in isposa, se mai tornasse vittorioso - fu condotto in una carcere. La Reina face a tutti palese che lo voleva morto, ma in questo mentre Laomedonte paleso che Euridamante era innocente et che fu calunniato dal principe Abante. 
Manifestata l'innocenza di Euridamante, e la scelleraggine di Abante, la giusta Reina volle meglio ubbidire alle leggi che alla Natura, e cosi diede ad Abante, suo figlio, quella pena, che meritava Euridamante se in vero fosse stato traditore. ${ }^{16}$

Il faut remarquer que l'évolution dramaturgique d'Élisabeth Moutzan - Martinengou - appuyée sur une formation uniquement livresque et théorique, puisque l'éventualité qu'elle ait elle-même assisté à quelque spectacle est pratiquement nulle ${ }^{17}$ - passe alternativement d'une première étape d'inexpérience, où le rôle déterminant a été joué, selon son propre aveu, par son inclination naturelle, à la recherche d'un certain nombre de codes dramaturgiques solides. Nous l'observons en train de ressentir de plus en plus intensément le besoin d'acquérir les connaissances théoriques jugées indispensables, se rapportant à des questions de distinction typologique, d'esthétique et de morphologie du texte théâtral.

Cette documentation théorique, qui, naturellement a été recherchée dans la bibliothèque familiale, ne peut qu'être dans sa majorité puisée dans les manuels classicisants d'esthétique qui circulaient en Europe au XvIIIe siècle et lors des premières décennies du XIXe. ${ }^{18} \mathrm{~L}$ 'un des principles inaltérables de la "bonne tragédie" demeure le respect aux règles aristotéliciennes, c'est-à-dire l'application attentive des trois unités classiques (lieu, temps et action). Certes, n'oublions pas que la possibilité de s'écarter de ces normes largement adoptées a sérieusement préoccupé les esprits au début du XIXe siècle. Mais il est peu probable que la noble zantiote, qui restait la plupart du temps enfermée chez elle et rencontrait peu de monde, ait été en état de saisir cette problématique -comme ce fut le cas d'Antoine Matessis, l'auteur du Basilic - et de recevoir les influences possibles de l'école romantique, ou encore, de s'aligner sur les rénovations esthétiques et idéologiques, déplaçant le focus de la pièce dramatique, innovations proposées par quelques représentants des Lumières, tel Diderot. Le circuit bien déterminé de ses sources d'information devait plutôt être dicté par le cadre de l'esprit académique de son temps. C'est ainsi que, parmi les principes fondamentaux qu'elle accepte pleinement, figurent la "fermeté" et le "pathétique" du caractère du héros tragique ainsi que la "moralité" du sujet traité.

Nous ignorons quels furent au juste les théoriciens dont elle a lu les ouvrages. Dans ce domaine un seul témoignage nous est parvenu, d'ailleurs fort suggestif en soi: Élisabeth avait à sa dispositon l'œuvre de Gian Vincenzo Gravina (1664-1718), Della Tragedia (1715). ${ }^{19}$ Gravina, jurinconsulte, 
philosophe et littérateur italien, a été considéré, à côté de Muratori et de G.B. Vico, comme l'un des penseurs-rénovateurs les plus importants de son siècle. De nombreuses affinités et simultanéités spirituelles le rapprochent notamment de Vico, qui, plus tard, allait développer en profondeur les théories de Gravina sur les liens existants entre la poésie et la philosophie. ${ }^{20}$ Retenons, en outre, que Gravina fut le maître et l'ami de Métastase. Parmi ses écrits, le plus célèbre en matière d'esthétique figure son traité Della Ragion poetica ( $\left.17^{08}\right)$, paru en deux volumes. Très répandu au cours du XVIIle siècle, il a même connu des traductions dans d'autres langues européennes. Parmi les fondateurs de l'Académie des Arcades, Gravina a rejeté l'esthétique du baroque et, orienté vers les sources de la poésie d'Homère et de Dante, s'est mis à la recherche de la pureté et de la simplicité de la "poésie ancienne" afin de retourner "à l'idée éternelle de la nature" (a un'idea eterna di natura), vers les principes d'une simple et pure raison ( $i$ principi di pura e semplice ragione) ${ }^{2 t}$ Une de ses idées maîtresses, devenant pour ainsi dire une des notions-piliers de sa théorie esthétique fut celle de délire (delirio), le résultat naturel de tout acte poétique; en effet, cette conception correspondait à la théorie préexistante de l'enthousiasme (furore), mais elle contenait un élément psychologique beaucoup plus fort; car, pour Gravina "la poesia" est "una maga ma salutare ed un delirio che sgombra le pazzie". ${ }^{22}$ Enfin, retenons le didactisme flagrant qui émane d'un autre concept central de Gravina: la poésie "si diletta e instruisce il popolo". ${ }^{23}$ Ses ouvrages théoriques ont contribué à enrichị la pensée du XVIIIe siècle européen, influençant des philosophes tels Locke, Montesquieu, Rousseau, ou encore dictant certains principes poétiques, réhabilités au début du XIXe siècle, par Ugo Foscolo par exemple. ${ }^{24}$ Il est enfin à noter que Gravina fut lui-même un auteur dramatique, ayant composé et ayant fait publier, en 1712, cinq tragédies: Palamede Andromeda, Appio Claudio, Papiniano et Servio Tulio.

Néanmoins, Élisabeth a également recherché, de son propre aveu, un appui théorique dans d'autres ouvrages d'esthétique; dans ce sens, il est possible qu'elle ait consulté, outre les quelques traités parus en langue italienne -des originaux ou des traductions- les manuels de Rhétorique publiés en grec à cette même époque par d'éminents adeptes des Lumières néohelléniques tels Néophyte Vamvas (Rhétorique, Paris, I813), Constantin Vardalachos (Rhétorique, Vienne, 1815) ou encore Constantin Oeconomos (Rhétorique, Vienne, ${ }_{18}{ }_{13}$, Grammatiká [= Poetique], Vienne, $\left.{ }_{18} 8_{17}\right) .{ }^{25}$ De plus, elle confia, en 182I, qu'en composant des drames tels que Evøú $\mu a \chi o \varsigma, E v$ - 


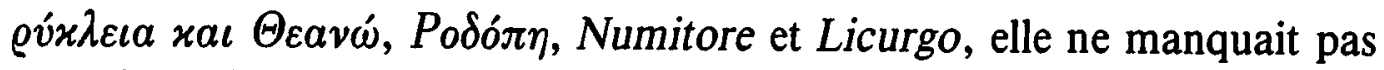
non plus d'étudier en même temps des écrivains qui enseignaient les règles de l'art tragique. ${ }^{26}$ Elle lisait également des tragédies et des drames dans le but non de copier thèmes et idées, mais parce qu'elle croyait que les passions envahiraient de cette manière plus impétueusement son cœur, ce qui lui permettrait à son tour d'inspirer des sentiments identiques à ses lecteurs. ${ }^{27}$ Cette dernière conception concernant l'excitement des passions, processus indispensable pour la création artistique, nous amème à rechercher des analogies avec les idées relatives exprimées par Grảvina, en ce qui concerne précisément les sources d'inspiration de l'acte poétique.

Il est remarquable que notre jeune écrivain zantiote ait cultivé avec beaucoup de zèle une grande variété de genres dramatiques, dont elle pose elle-même la différenciation typologique, puisqu'elle se réfère à eux dinstinctement; drames, tragédies, tragédies morales, etc. D'ailleurs, la meilleure preuve de son vif intérêt pour les questions de théorie esthétique est démontrée par sa tentative de rédiger une Poétique, oeuvre qui devait certainement s'appuyer sur ses lectures réciproques. ${ }^{28}$

Or, pendant cinq ans (1820-1825), Élisabeth écrivit sans interruption; il en résulta vingt-deux pièces. ${ }^{29}$ Selon ses dires, son rythme ralentissait seulement au cours des grandes chaleurs d'été. C'est alors qu'elle s'adonnait à des compositionș plus légères, notamment poétiques. Toutefois, c'est le genre dramatique; un travail qui demande beaucoup de discipline et de concentration, qui semble l'émouvoir davantage. ${ }^{30}$

Vingt-deux pièces: un bilan remarquable. Parmi les titres, certains peuvent nous conduire à quelques hypothèses en ce qui concerne les influences possibles qui s'y exercent. Élisabeth déclare d'ailleurs elle-même avoir étudié très assidûment tous les écrivains dramatiques dont les ouvres existaient dans la bibliothèque paternelle.

Néanmoins, la référence à deux titres présente un intérêt plus spécial. Il s'agit, en premier lieu, de la tragédie Bruto primo, rédigée en langue italienne, qui nous fait penser à l'œeuvre bien connue de Vittorio Alfieri; c'est à cette même source d'inspiration qui nous conduit également le second titre, II Tiranno punito. ${ }^{31}$ Les deux pièces ont été conçues et terminées presque simultanément. Nous ne pouvons donc pas omettre la possibilité que la seconde pièce ait également exploité la filière des motifs puisés dans l'histoire de la Rome antique et, en particulier, dans la Mort de César, un des sujets favoris de la dramaturgie "républicaine" 
des Lumières. C'est ainsi que s'esquissent quelques traces du théâtre politique et militant des Lumières (Voltaire, Alfieri). ${ }^{32}$

Les pièces citées dans son Autobiographie ne coîncident pas tout à fait avec celles mentionnées dans le Catalogue sommaire de ses ouvrages ( $K \alpha$ $\tau a ́ \lambda o \gamma o \varsigma \tau \omega v \Sigma v \gamma \gamma \varrho a \mu \mu a ́ \tau \omega v \tau \eta \varsigma)$ joint par son fils unique Elissavétios à l'édition posthume de son Autobiographie. ${ }^{33}$ Le Catalogue ne contient pas des titres certains que nous repérons cependant dans son texte autobiographique, tandis qu'on y trouve d'autres titres qui ne figurent pas dans le texte mentionné ci-dessus.

\section{CATALOGUE SOMMAIRE}

(Le chiffre arabe entre parenthèses renvoie à l'édition de l'Autobiographie que nous avons utilisée)
(Titres ajoutés, puisés directement dans l'Autobiographie de l'auteur)

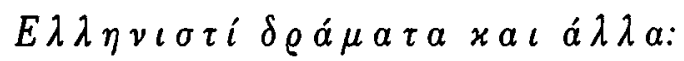

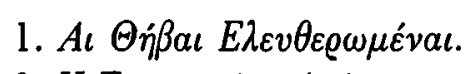

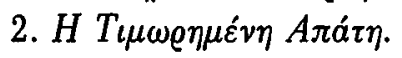

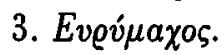

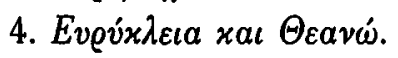

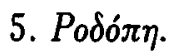

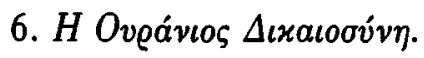

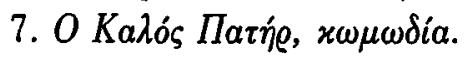

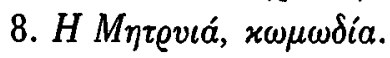

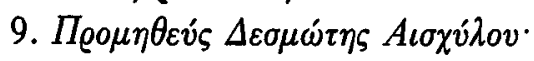
$\alpha \pi \lambda \sigma \varepsilon \lambda \lambda \eta \nu \iota x \dot{\eta} \mu \varepsilon \tau \dot{\alpha} \varphi \varrho \alpha \sigma \iota \varsigma$ $\varepsilon \iota \varsigma \pi \varepsilon \zeta o ́ v$.

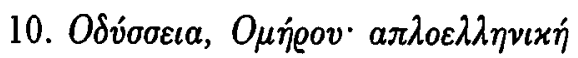

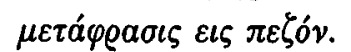

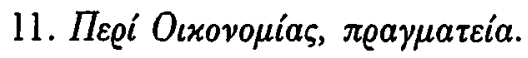

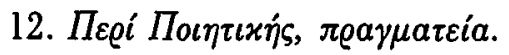

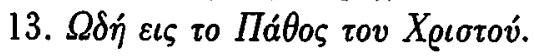

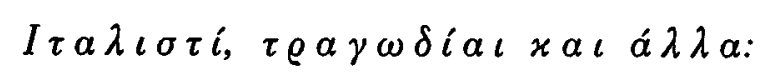

1. Numitore, tragedia.

2. Bruto primo, tragedia.

3. Enrico o l'Innocenza, tragedia immaginaria.

4. Laodice o la Prudenza, tragedia morale.

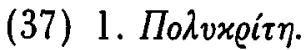

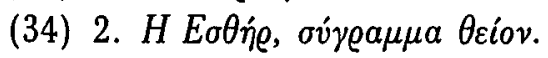

(34) 3. $\Sigma \dot{\varepsilon} \sigma \omega \sigma \tau \iota \iota$.

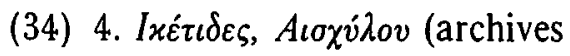
Sigouros, cf. BOUBOULIDIS, p. $\left(\theta^{\prime}\right)$ 
5. Il Tiranno punito, dramma morale.

6. La Teano o la giustizia legale, dramma.

7. Licurgo o la Mansuetudine, dramma morale.

8. Gil effetti della discordia, tragedia morale.

9. L'Eccellenza della giustizia, dramma.

10. Il Rigore delle Leggi, tragedia.

11. Il buon re, dramma morale.

12. Libro di diverse composizione poetiche.

13. I Pastori [amici?], commedia morale in un atto.

14. Novelle morali.

15. La Saggia madamigelia, commedia.

16. Il buon procuratore, commedia. $^{34}$

Il est tout à fait clair que, dans cet inventaire panoramique de l'abondante production d'Élisabeth Moutzan-Martinengou, les motifs prédominants sont en corrélation étroite avec les revendications de la dramaturgie néoclassique, qui impose que les sujets soient recherchés dans une variété de thèmes mythologiques et historiques. En outre, le souci moralisant et didactique qui semble être prépondérant ici s'harmonise parfaitement avec les conceptions correspondantes qu'ont cultivées les Lumières européennes et dont on retrouve les répercussions de façon persistante au tournant du XVIIIe siècle chez les adeptes du mouvement des Lumières en Grèce.

Aucune de ses pièces s'inscrivant dans le genre sérieux n'est arrivée jusqu'à nous. Nous fondant uniquement sur un nombre minime de données indirectes, nous ne pouvons donc formuler que quelques hypothèses ou encore proposer des conclusions générales. En revanche, dans le domaine du genre comique, une de ses dernières pièces du point de vue chronologique,

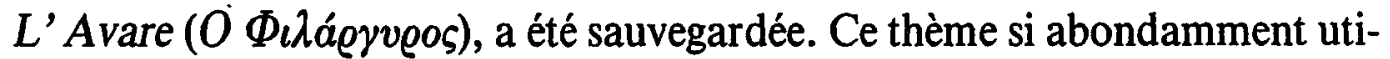
lisé dans la dramaturgie depuis les Anciens appartient évidemment à une longue et fertile tradition, qui a inspiré plusieurs auteurs comiques, notamment Molière et Goldoni pour nous limiter aux temps modernes. Il faut également 
prendre en considération le fait que, lorsque l'idée vint à Élisabeth Moutzan s'occuper de manière créative du caractère de l'avare, la célèbre adaptation de Constantin Oeconomos avait déjà circulé en grec. ${ }^{35}$ Sans doute, cette œuuvre qui visa à exprimer certains desiderata idéologiques des Lumières néohelléniques, n'est-elle pas été passée inaperçue dans le contexte culturel pré-révolutionnaire. Dans son importante Préface adressée aux Grecs (Прos

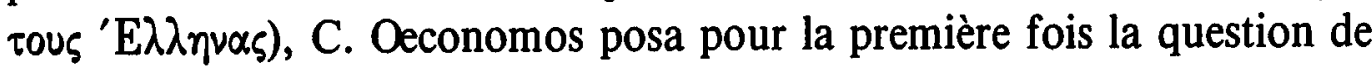
la comédie nationale et plaida en sa faveur. Il argumenta en ce que concerne le besoin urgent d'attribuer au comique un habit national car, dit-il, c'est uniquement par cette voie que nous réussirons à corriger efficacement les mours. Il est vrai que C. Oeconomos n'a formulé à l'usage et au profit du public grec que des conceptions au sujet de la création d'une comédie nationale très répandues dans l'Europe du XVIIIe siècle et soutenues avec persistance par des penseurs tels que Hugh Blair ${ }^{36}$

L'Avare d'Élisabeth Moutzan-Martinengou s'intègre de manière plus ou moins consciente à cette tradition: la pièce propose en effet un tableau lucide de quelques spécificités typiquement heptanésiennes et renferme à maints niveaux des éléments de critique sociale. Quoique sur le plan proprement dramaturgique cette pièce présente, et ceci est justifiable, des imperfections, elle réussit, en revanche, à créer une image véridique de la vie domestique zantiote du début du XIXe siècle, tandis que de nombreuses allusions d'un caractère plus général à un renouveau politique, marqué par des efforts de modernisation bourgeoise, permettent des projections sur les structures sociales heptanésiennes durant cette période de transition du. système féodal périmé, hérité des Vénitiens.

Malgré son inexpérience, Élisabeth y créa des caractères intéressants. ${ }^{37}$ Le héros principal Sélimos ( $\Sigma \dot{\prime} \lambda \eta \mu o \varsigma$ ) possède de qualités nocives similaires

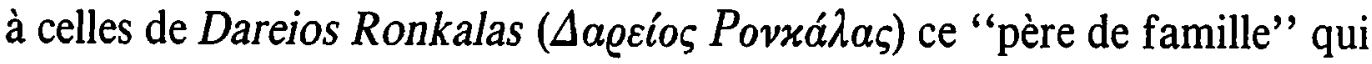
est le personnage central du Basilic d' Antoine Matessis. ${ }^{36}$ Sélimos est peint comme un caractère rude et autoritaire qui impose cruellement son pouvoir aux membres de sa famille. Sa présence pèse lourdement sur les siens et étouffe son entourage. La démonstration exagérée de son avarice ne semble ni créer d'effets comiques, ni provoquer le rire. C'est ainsi que l'élément purement comique, voire burlesque de la pièce, n'apparaît que dans les scènes secondaires, attribué notamment au comportement des serviteurs. Il est, en outre, très intéressant de noter que dans l'Avare, tel qu'ilaété conçu par la jeune zantiote, est totalement absent un motif répandu dans les pièces précédentes, celui du vieillard amouraché d'une femme beaucoup plus jeune 
que lui et désirant l'épouser. Ridiculisant davantage le personnage de l'avare, cet aspect de l'intrigue allégeait sensiblement la gravité de son défaut. Sélimos, non accompagné de ces stéréotypes burlesques appartenant aux traditions comiques antérieures, est unilatéral, entouré de ses proches mais profondément solitaire, presqu'ascétique autant que cruel.

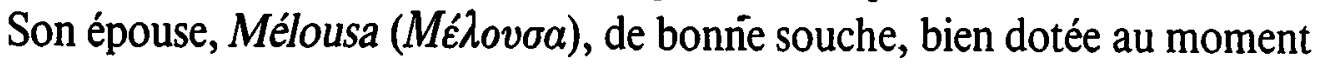
de son mariage est une esquisse du type de la noble zantiote, retirée, soumise et vivant sous le pouvoir despotique de son mari. Son monologue introductif à la comédie est de ce point de vue éloquent: ${ }^{39}$

\author{
ACte Premier \\ $\mathrm{S} \mathrm{c}$ ène I \\ La chambre à coucher de Mélousa. \\ Mélousa étale du linge sur une table. \\ Puis entre Tylessa
}

MÉL: Oh, que je suis malheureuse! Je me sens épuisée en étalant ce linge (elle laisse à côté le fer à repasser)... Est-il jamais possible qu'une femme de mon état, ayant reçu une dot pareille, ayant tant de biens, puisse faire ce genre de travail? Que le Ciel brûle le négociateur de ce mariage, que le Ciel brûle celui qui s'était entremis pour que j'entre ici et que j'épouse un homme aussi avare. Hélas! A partir du moment que je l'ai épousé, je ne me suis plus amusée, je n'ai pris aucun plaisir, ascun divertissement, aucun petit délice; je ne reçois que des insultes, des embarras et des blâmes concernant ce que je mange, ce que je bois, ou encore ce que je porte. Ah! quelle infortune! Quand est-ce que moi, je ne parle pas d'aujourd'hui où je suis devenue presque une vieille femme, mais du temps où j'étais jeune, j'ai fait faire une belle robe? Quand est-ce que je suis sortie de chez moi bien parée ainsi qu'il convenait à mon rang?... Mais pourquoi est-ce que je pense à tout cela?... Hélas?... Tout ce que je viens de dire n'est rien devant ce qui suivra. Lui, de peur de dépenser de l'argent, il a laissé notre fils unique sans convenable éducation, ${ }^{40}$ et je verrai mon fils prendre très vite le mauvais chemin car l'avarice de mon mari, le jetant dans la débauche, sera le cause de sa perte. Lui, craignant que je ne lui demande un sou pour faire quelque aumône, ne supporte même pas le prêtre chez nous... Oh! malheur à moi!... Vivant près de cet espèce de chien, j'ai détruit, pour ainsi dire, ma vie et je risque de perdre mon âme... Mais non, non! Cela ne peut pas se faire. Je ne suis pas vendue à lui comme une esclave... Je suis seule au monde, je n'ai aucun proche mais je possède tant de maisons, tant de biens que rien ne me manquera... Le jour de ta punition viendra, très méchant Sélimos. Mes droits sont puissants et aucune force ni extérieure ni intérieure ne pourra les repousser (Elle prend le fer et commence à repasser, mais tout de suite elle le met à côté)... Je l'ai oublié et il s'est refroidi... Tylessa! Tylessa! ${ }^{4}$ 
Dans la peinture sombre de ce type féminin extrêmement oppressé sont rassemblés tous les jugements personnels d'Élisabeth à l'égard de la position sociale de la femme zantiote éparpillés dans son Autobiographie. Mélousa apparaît profondément résignée; elle se révolte seulement lorsque tout tourne au pire, lorsque précisément son fils unique, Lékaos (1éxaos), jeté par son père en prison, menace de se suicider. C'est alors que Mélousa prend la décision de rompre son esclavage, d'abandonner sa maison et de se réfugier au couvent. ${ }^{+2}$

Or, dans cette pièce, un nouvel ordre de choses est esquissé. Mélousa -le choix du prénom contient peut-être quelques allusions symboliques à ce qui est réservé au futur $(=\mu \varepsilon ́ \hat{\lambda} \lambda \circ v)$ - réussira à s'éloigner de l'autorité de son époux, Sélimos sera puni doublement pour son vice, perdant tout d'abord son trésor, puis perdant sa famille. Enfin, dans cette œuvre qui critique l'ancien état des choses - Sélimos est l'ultime représentant d'un système pourri- de nouveaux types sociaux apparaissent, s'élèvent contre

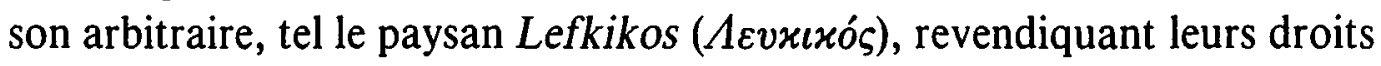
et mettant fin à sa tyrannie de type féodal.

SÉL.: Ah, ce Tribunal-là, ce Tribunal, combien de mal me provoque-t-il! O temps passés, où êtes-vous! O temps de jadis, temps dorés pour moi, bienheureux, où êtes-vous allés! Ah! pauvre Provéditeur [Gouverneur], pauvre Provéditeur, Provéditeur!... A l'époque, c'était la force qui tenait la première place à Zante. Désormais, c'est la loi. Le jugement est attribué à quiconque le mérite. Hélas, aucune faveur n'est plus accordée au riche ou au noble $[\ldots]^{43}$

Du point de vue idéologique, cette comédie de caractère dont l'auteur réhabilite adroitement maints éléments et qui constitue un véritable tableal des moeurs zantiotes de son temps, contient, d'une part, quelques germes d'une critique ressentie contre l'ancien mode de vie et son système de valeurs et, d'autre part, l'espérance en la création d'une ère nouvelle, émancipée de tous les préjugés rétrogrades tant moraux que sociaux. Il est clair que l'unique pièce sauvegardée d'Élisabeth Moutzan-Martinengou porte le vent rénovateur des Lumières.

\section{$\sum \eta \mu \varepsilon \iota \dot{\omega} \sigma \varepsilon \iota \zeta$}

I. Phédon C. Bouboulidis, Elisabeth

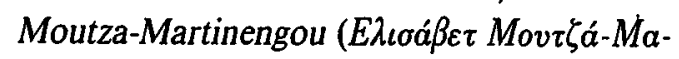

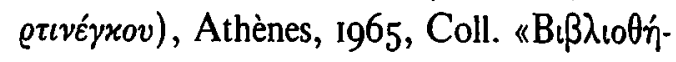

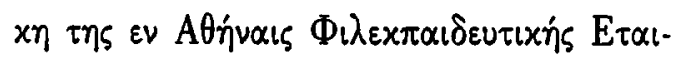

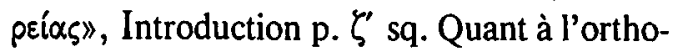
graphe du nom de famille, $M$. Bouboulidis a 
a utilisé le type Moutza, tandis que d'autres chercheurs préfèrent l'orthographe Moutzan, attestée, selon eux, dans des documents heptanésiens. Toutes les citations concernant le texte autobiographique d'Élisabeth MOUTZAN-MARTINENGOU seront puisées dans l'édition critique de $\mathrm{Ph}$. Bouboulidis (op. cit.). Il existe également une traduction anglaise effectuée par Helen DENDRINOUKOLIAS; voir Élizabeth MOUTZAN-MARTINEN. GOU, My Story, publié par les soins de Helen DENDRINOU-Kolias, Athènes, Georgia U.P., 1989.

2. Nous devons aux soins du fils unique de l'auteur la première édition posthume de l'œuvre, publiée sous le titre; Ma Mère, autobiographie de Madame Élisabeth MoutzanMartinengou, éditée par son fils Élissavétios Martinengos, suivie de quelques poésies dive-

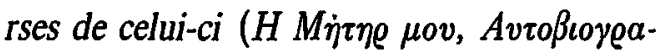

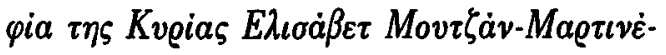

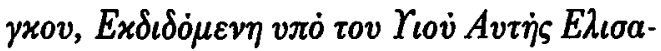

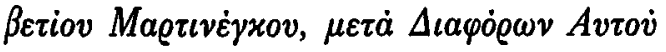

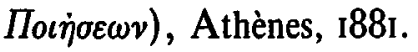

3. C. Th. Dimaras, Histoire de la

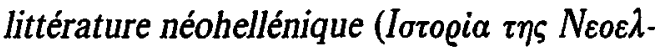

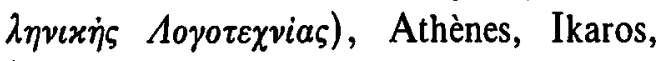
${ }^{6}$ I975, p. 216 sq.

4. Voir à ce sujet Paschalis M. KitromiLIDES, "The Enlightenment and Womanhood: Cultural Change and the Politics of Exclusion" Journal of Modern Greek Studies, vol. I, No 1 (May 1983), pp. 56-6r.

5. Cf. l'article rédigé par Néta APoStolidou, Dictionnaire Biographique Univer-

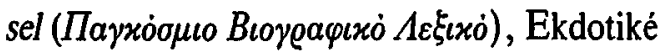
Athinôn, vol. vI, pp. 287-88. Une approche interprétative récente de l'Autobiographie a été proposée par Georges D. Kalogeras, "Éditions et Approches d'une Vie: L'Autobiographie d'Élisabeth Moutzan-Martinengou"' (Ex-

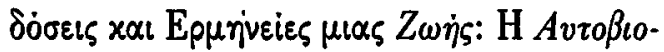

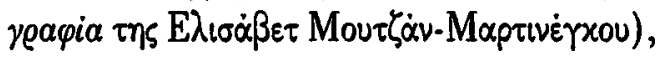

$\Gamma \varrho \dot{\alpha} \mu \mu a / G r a m m a, ~ I ~(1993)$, pp. 63-85.

6. Phédon C. Bouboulidis, op. cit, p. 3 . 7. Op. cit., p. 13 sq.

8. Ibid., p. 7 sq.

9. Phédon C. Bouboulidis, op.cit., p. 5 . 10. Op. cit., pp. $35-36$.

II. Plusieurs éditions de la traduction en grec de cette œuvre avaient circulé au début du XIXe siècle.

12. Cf. Anna TabaKi, "Les conceptions pédagogiques dans la traduction grecque $\mathrm{du}$ Traité des études de Charles Rollin" $(\mathrm{O} \iota \pi \mathrm{\pi}$ -

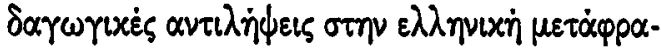

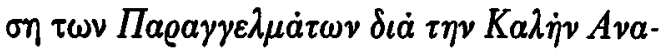

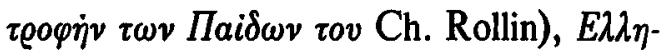
vıxá, 45(I995) I, pp. 75-84 (avec un résumé en anglais). Une version française de cette étude a paru dans le Bulletin de Liaison no I3, Centre d'Études Balkaniques, INALCO, décembre I995, pp. 33-46 (traduction Henri TONNET).

13. Nous mentionnons également les Eléments de Logique et de Philosophie Mo-

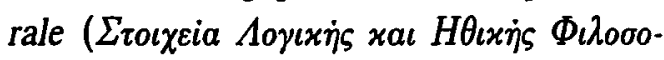
yias, 1808) de HEINECKE, traduits par Grégoire Brânconeanu-Basarab. Aussi, dans le but de s'exercer, Élisabeth a-t-elle entrepris la traduction des Novelle de Francesco SOAvE et les Dialogues des Morts de Lucien.

I4. Phédon C. Bouboulidis, op. cit., p.

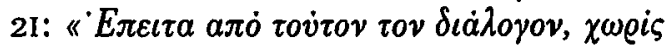

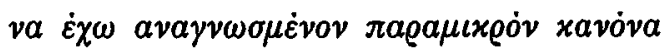

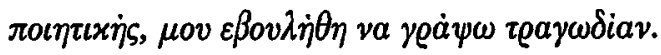

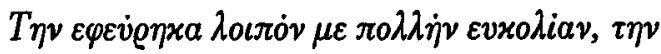

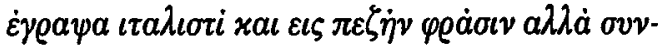

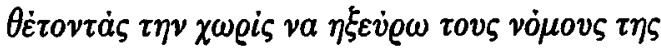

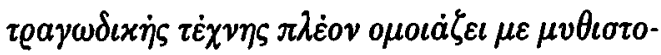

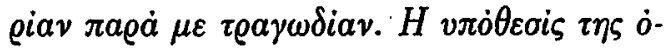
$\mu \omega \varsigma-\eta$ oлoia $\varepsilon \iota \varsigma$ to $\pi \iota \theta a v \dot{o} \nu \varepsilon \theta \varepsilon$ -

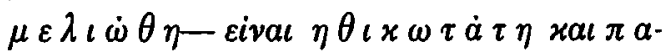
$\theta \eta \tau \iota x \omega \tau \dot{\alpha} \tau \eta$, $\omega \sigma \dot{\alpha} v$ ojov $a \pi \delta \delta \varepsilon i x \nu \varepsilon \iota \dot{\sigma} \tau \iota$

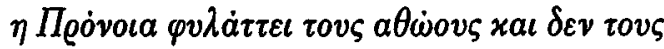

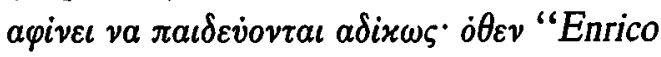




\section{L'OEUVRE DRAMATIQUE D'ÉLISABETH MOUTZAN-MARTINENGOU}

ol 'innocenza" $\tau \eta v \varepsilon \pi \omega \nu \dot{\mu} \mu \alpha \sigma a »$. C'est moi qui souligne.

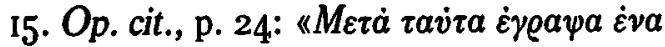

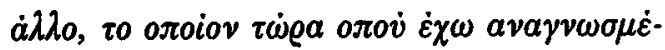

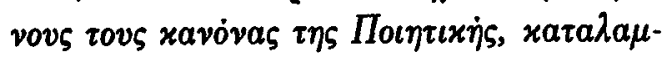

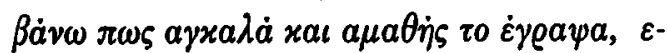

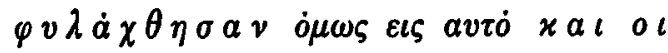
$\tau \varrho \varepsilon \iota \varsigma \varepsilon \nu \dot{\nu} \tau \eta \tau \varepsilon \varsigma, \quad \dot{\eta} \gamma o v \nu \eta \varepsilon \nu \dot{o} \tau \eta \zeta$ $\tau \eta \varsigma \pi \varrho \dot{a} \xi \varepsilon \omega \varsigma, \eta \varepsilon v \dot{\partial} \tau \eta \varsigma \tau 0 v x a \ell-$ $\varrho \circ \dot{v} x a \imath \eta \varepsilon v \dot{0} \tau \eta \varsigma \tau \sigma v \tau \dot{0} \pi 0 v$. Els

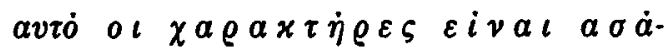
$\lambda \varepsilon v \tau o \iota, \varepsilon \iota \varsigma a v \tau \dot{\delta} \tau 0 \pi a \theta \eta \tau \iota x \dot{o} v$ $x a \iota \tau o \eta \theta \iota x \dot{\delta} v \pi \circ \lambda \dot{v} \varepsilon \mu \beta a i v \varepsilon \iota x a l$,

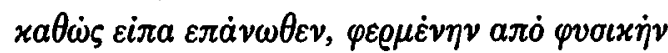

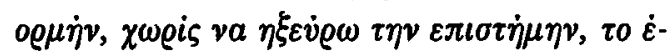

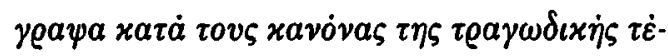
$\chi^{\nu \eta} \varsigma^{»}$. C'est moi qui souligne.

I6. Op. cit., p. 25.

17. Sur l'ambiance dramaturgique heptanésienne, voir Anna TABAKI, Le théâtre néohellénique, op. cit., vol. I, chap. II et III , p. I05 sq., où l'on peut trouver la bibliographie relative à se sujet. Voir aussi, D. Rommas, "Le théâtre heptanésien" "To

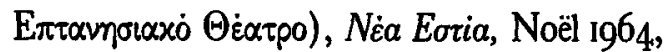
pp. 97-167; Glykerie Protopapa-BoubouLIDOU, Le théâtre à Zante du XvIIe au XIXe

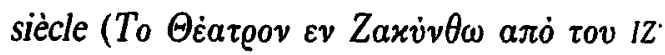

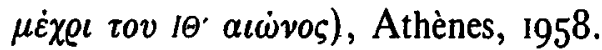

I8. Parmi les manuels européens d'esthétique qui étaient assez répandus dans le monde culturel grec, nous devons citer les Principes de Littérature de Charles Batteux, le Lycée de LAHARPE ainsi que les Lectures on Rhetoric and Belles Lettres de Hugh BLAIR, ce dernier ayant pénétré dans le circuit grec par le biais de sa traduction française (première édition, Paris, 1808). Ajoutons que les ouvrages de trois auteurs mentionnés ont connu des traductions en italien, ce qui augmente sensiblement la possibilité de leur existence dans la bibliothèque zantiote des Moutzan.

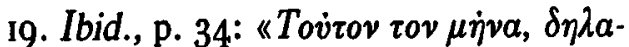

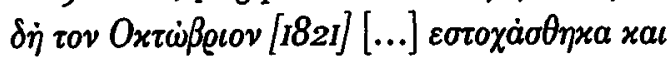

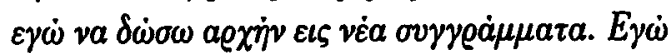
$\tau \dot{\tau} \tau \varepsilon \varepsilon i \chi \alpha \alpha \nu \alpha \gamma \nu \omega \sigma \mu \dot{\varepsilon} v \eta v \tau \eta \nu \mathrm{H} \theta i x \dot{\eta} \nu \Phi_{l} \lambda 0 \sigma 0$ -

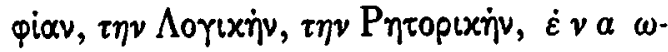
$\varrho a i o \sigma \dot{v} \gamma \gamma \varrho a \mu \mu a \tau o v \Gamma \varrho a \beta i v a, 0$ $\pi \circ \dot{v} \delta \iota \delta \dot{a} \sigma x \varepsilon \iota \tau \eta v \tau \dot{\varepsilon} \chi \nu \eta v \tau \eta$ $\tau \varrho a \gamma \omega \delta i \alpha \varsigma[. .] »$. C'est moi qui souligne.

20. Voir Wladyslaw Tatarkiewich, History of Aesthetics, vol. III Modern Aesthetics, Warszawa, Mouton, 1974, p. 444. Aussi Michael Mooney, Vico in the tradition of Rhetoric, Princeton, New Jersey, Princeton University Press, 1985, pp. 147-149. Cf. Amedo Quondam, Cultura e ideologia di Gian Vincenzo Gravina, Milano, Mursia, 1968, Filosofia della luce e luminosi nelle Egloghe dell Gravina: Documenti per un capitolo della cultura filosofica di fine Seicento, Studi Vichiani, no 3, Napoli, Guida, 1970.

2I. Voir Laffont-Bompiani, Dictionnaire des œuvres, Iv, p. 207. Aussi, Enciclopedia Italiana di Scienze, Lettere ed Arti, McmXXXII-XI (1932-I94I).

22. Voir Wladyslaw Tatarkiewich, op. cit., p. 443 sq., pour la citation ci-dessus, p. $44^{8 .}$

23. Op. cit., p. 449.

24. Enciclopedia Italiana di Scienze, Lettere ed Arti, op. cit.

25. En ce qui concerne la pénétration d'un certain nombre de concepts esthétiques nouveaux dans le monde culturel grec ainsi que l'impact dans l'aire hellénique so'ds domination ottomane de quelques théories européennes en vogue à travers justement les ouvrages mentionnés, voir l'étude d'Athanasie GLYKOFRYDI-LÉONTSINI, L'Esthétique néohel-

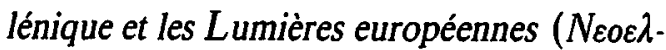

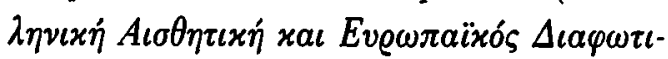
o $\mu o ́ \varsigma)$, Athènes, 1989.

26. Elle nous confesse que, lorsque' elle 
étudiait par exemple le Decamerone de BocCACE, elle a été grandement secourue par l'oeuvre de Salv. Corticelli, Discorsi sulla toscana eloquenza (Bologna, 1752) afin de résoudre les difficultés stylistiques du texte. Voir Phédon C. Bouboulidis, op. cit., p. 39.

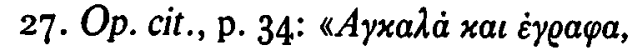

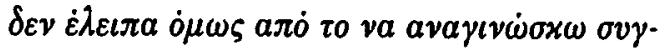

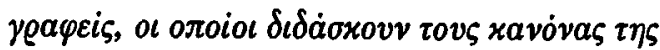

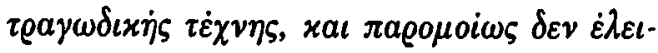

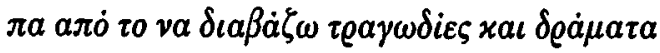

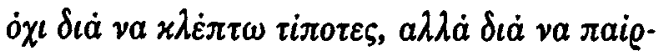

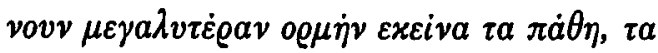

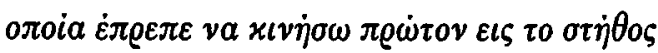

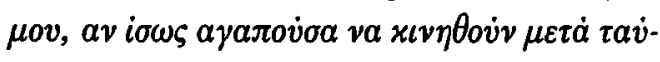

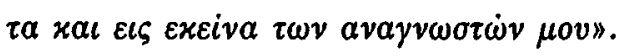

28. Phédon C. Bouboulidis, op. cit., p. 157.

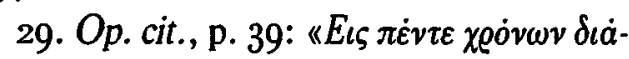

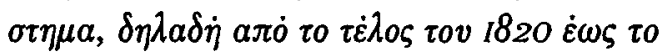

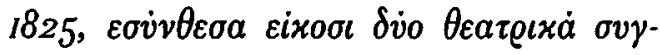

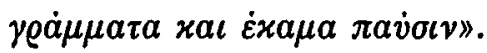

30. Ibid., p. 37 .

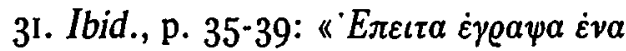

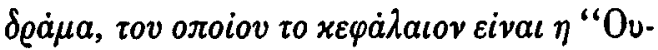

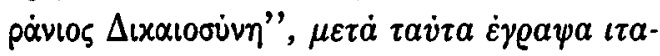

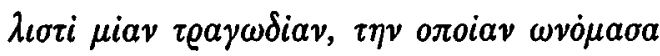

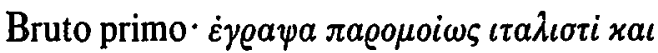

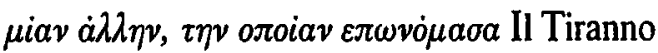

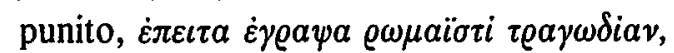

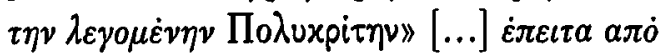

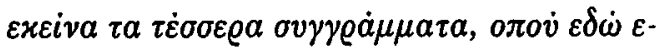
$\pi \dot{\alpha} \nu \omega \varepsilon \sigma \eta \mu \varepsilon i \omega \sigma \alpha, \varepsilon \sigma \dot{v} v \theta \varepsilon \sigma \alpha \varkappa \alpha \iota \dot{\alpha} \lambda \lambda \alpha \delta \dot{v} o, \tau \alpha$

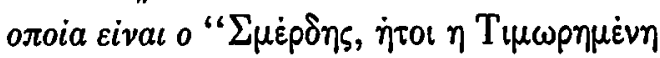

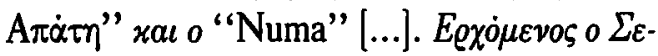

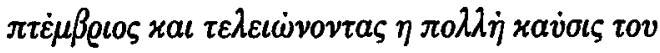

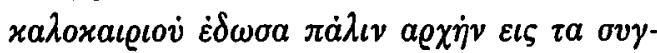

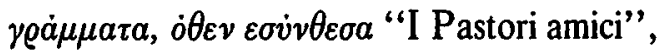

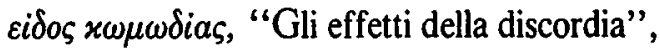

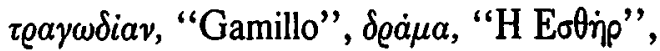

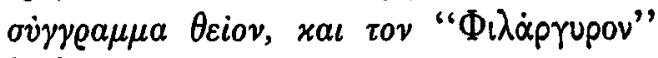

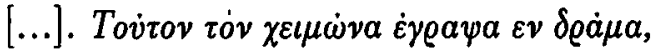

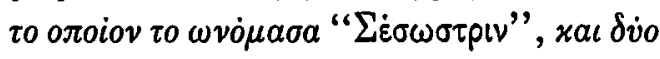

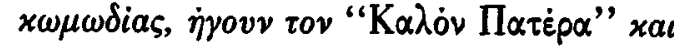

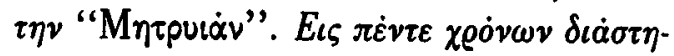

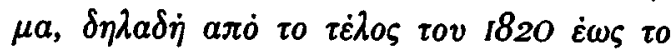

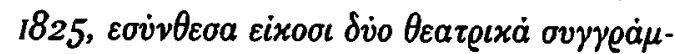

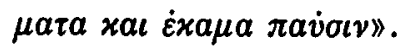

32. En ce qui concerne la réception du théâtre dit de propagande philosophique dans la dramaturgie néohellénique, je me permets de renvoyer à mon étude "La résonance des idées révolutionnaires dans le théâtre grec des Lumières (1800)-1821)", Actes du IIle Colloqne d'Histoire du Centre de Recherches Néohelléniques "La Révolution française et l'Hellénisme moderne" (Athènes, I4-17 octobre 1987), Athènes, CRN/FNRS, 1989, pp. 471-490. Cf. la monographie de Z. SiAFLEKIS, Le glaive et la pourpre. Le tyrannicide dans le théatre moderne. Aix-en-Provence, Edisud, 1984 .

33. Elissavétios Martinengos, Ma Mère, autobiographie de Madame Élisabeth Moutzan-Martinengou, op. cit., Athènes, $188 \mathrm{I}$.

34. Phédon C. Bouboulidis, op. cit., pp. 157-158.

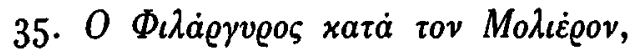
Vienne, 1816.

$3^{6 .}$ Cf. l'analyse exhaustive de la question, que j'ai opérée dans mon article "Approches de l'œuvre moliéresque au xixe siècle en

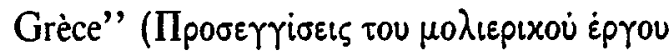

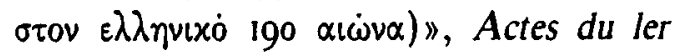
Congrès International de l'Association Grecque de Littérature Générale et Comparée. Rapports de la littérature grecque avec les

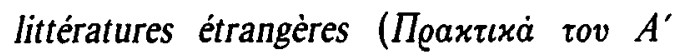

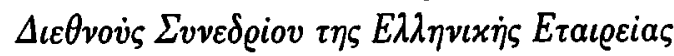

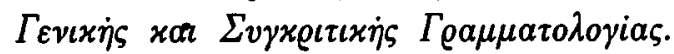

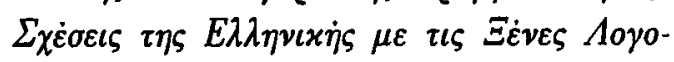
$\tau \varepsilon \chi v i \varepsilon \varsigma)$, Athènes, Domos, 1995, pp. 367 -385 .

37. Phédon C. Bouboulidis, op. cit., p. $\zeta$, remarque lui aussi tant la peinture de caractères intéressants que les germes de 


\section{L'OEUVRE DRAMATIQUE D'ÉLISABETH MOUTZAN-MARTINENGOU}

critique sociale sous-jacents dans la pièce.

38. Évidemment et malgré l'importance attribué à ce personnage dramatique par la plume de ces deux heptanésiens, à savoir Élisabeth Moutzan-Martinengou et Ant. Matessis - ce dernier ayant très probablement reçu l'influence de Diderot- l'hypothèse d'une source commune dans les deux cas, celle de la pensée de Diderot, serait très osée et non argumentée.

39. Je suis tentée de rapprocher l'adoption de cette solution, qui introduit bien des éléments cruciaux pour le développement de l'intrigue de la pièce, de l'initiative similaire de C. Oeconomos dans sa propre adaptation, qui débute par un monologue de Zoitza (Élise dans la pièce moliéresque), entièrement inventé et ajouté par l'auteur.

40. Le même motif du manque d'éducation du fils de l'Avare est également présent dans l'adaptation de $C$. Oeconomos.

41. Le texte grec est le suivant:

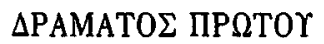

\section{$\Sigma$ KHNH A}

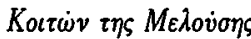

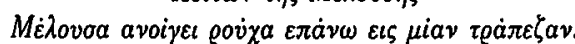

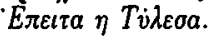

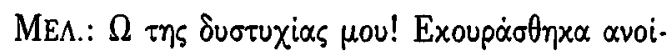

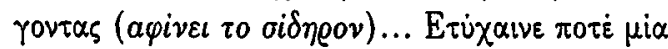

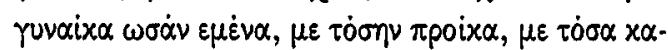

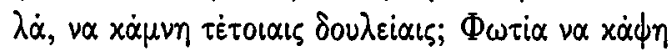

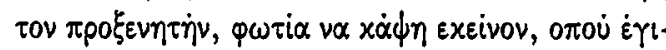
vEv $\alpha \varphi O p \mu \dot{\eta} v \alpha \dot{\varepsilon} \mu \pi \omega \varepsilon \gamma \dot{\omega} \dot{\omega} \delta \dot{\omega} \mu \dot{\varepsilon} \sigma \alpha, \nu \alpha \lambda \dot{\alpha} \dot{\beta} \omega \delta i \dot{\alpha}$

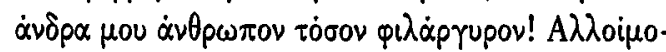
vov! A

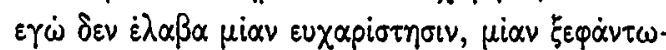

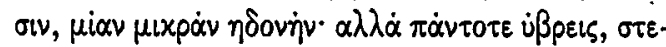

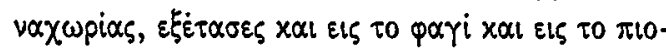

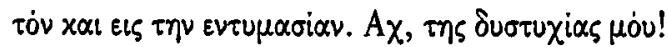

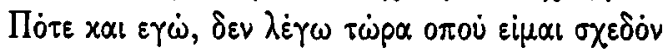

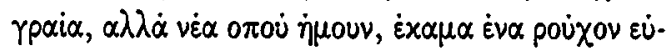

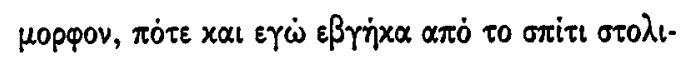

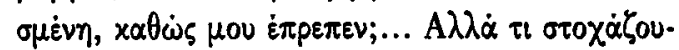

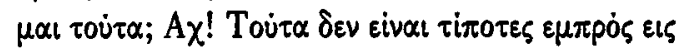

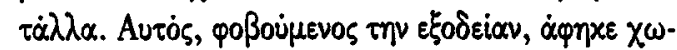

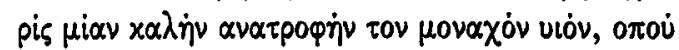

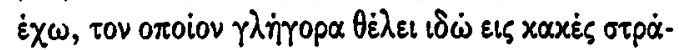

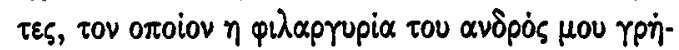

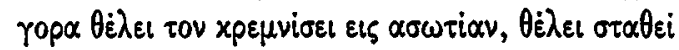

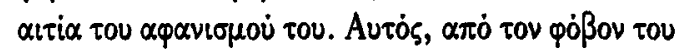

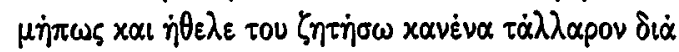

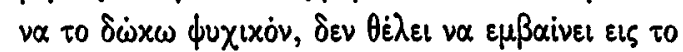

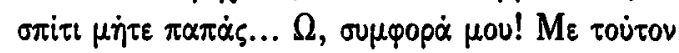

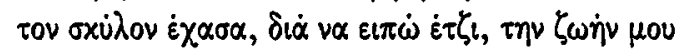

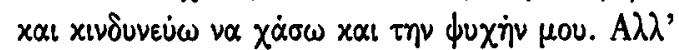

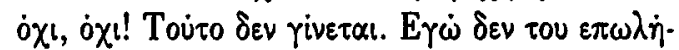

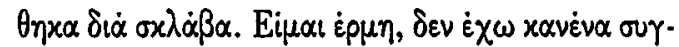

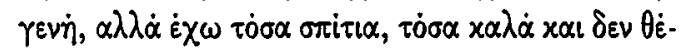

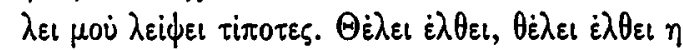

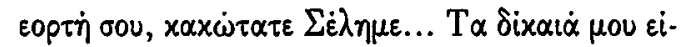

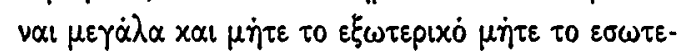

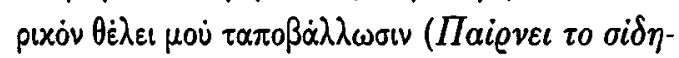

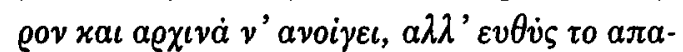

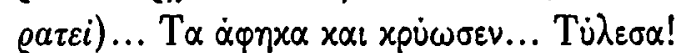
Tủ $\varepsilon \sigma \alpha$ ! Voir Phédon C. Bouboulidis, op. cit., pp. 6I-62.

42. Pour l'auteur, cet acte constitue décidément un acte de libération; l'idée utopique qu'elle se fait dans son Autobiographie de la vie religieuse dans les couvents, réservée à la paix de l'exercice spirituel, la possède et demeure pour longtemps son propre rêve; cf BouBoulidis, op. cit., pp. II-12, ainsi que la note de la page 55 .

45. Le texte grec est le suivant:

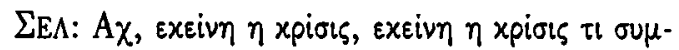

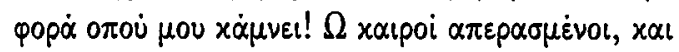

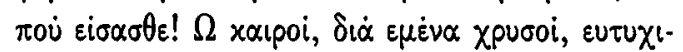

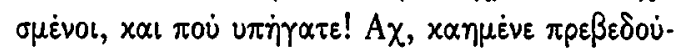

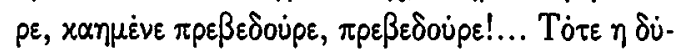

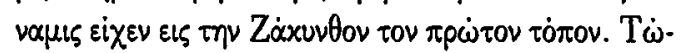

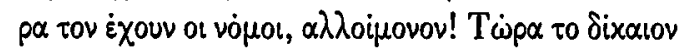

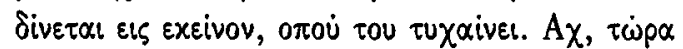

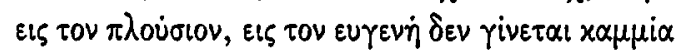
$\chi \dot{\alpha}$ pis! [...]. Voir Phédon C. Bouboulidis, op. cit., p. 90. 


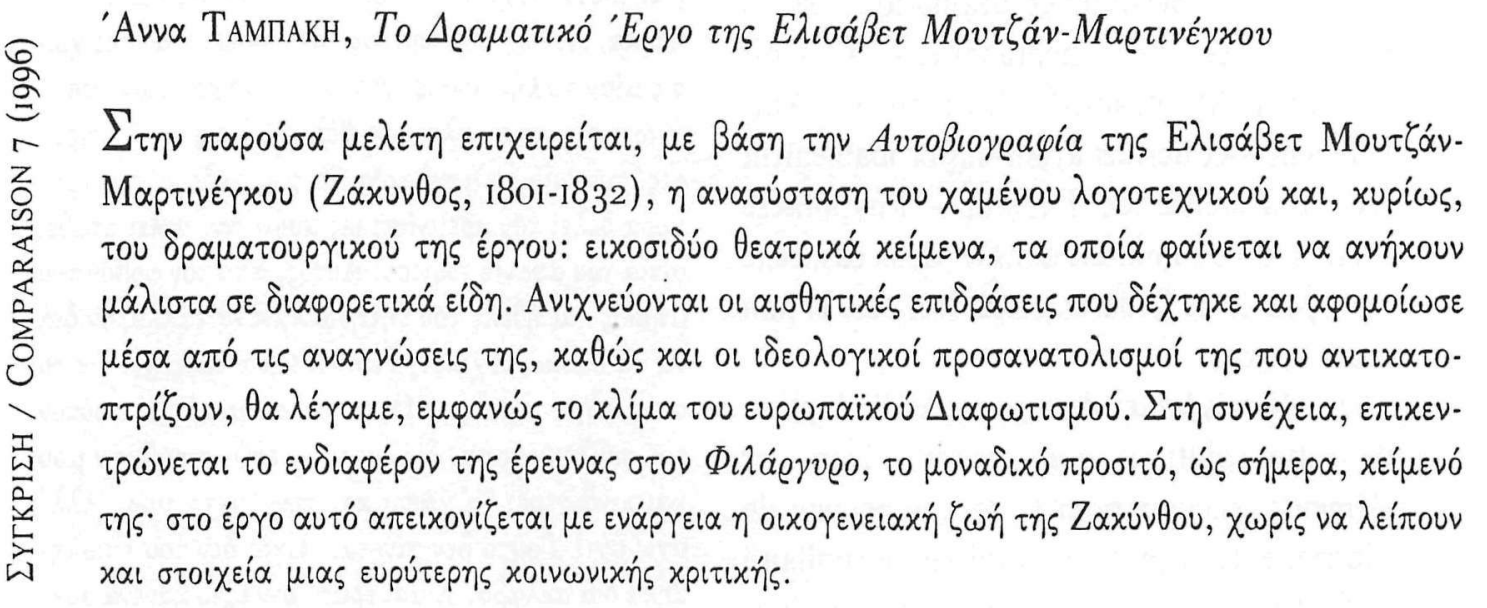

